ISSN 0103-8478

\title{
Competitividade de capim-pé-de-galinha com soja
}

\author{
Competitiveness of goosegrass with soybean
}

\begin{abstract}
Alana Cristina Dorneles Wandscheer ${ }^{I^{*}}$ Mauro Antônio Rizzardi ${ }^{\mathrm{I}}$ Muriel Reichert ${ }^{\mathrm{I}}$ Fernando Gaviraghi ${ }^{\mathrm{I}}$
\end{abstract}

\section{RESUMO}

A competição é uma interferência negativa, na qual indivíduos competem por recursos do meio. Objetivou-se avaliar a competitividade do capim-pé-de-galinha com soja. O experimento foi realizado em casa-de-vegetação, em delineamento inteiramente casualizado e quatro repetições. Os tratamentos foram cinco proporções da cultura e da espécie daninha, respectivamente, 8:0, 6:2, 4:4, 2:6 e 0:8, que correspondeu a 100, 75, 50, 25 e $0 \%$ de plantas de soja, e o inverso para o capim-pé-de-galinha, com população final constante de oito plantas vaso-1 $(8 \mathrm{~L})$, correspondendo a aproximadamente 250 plantas $\mathrm{m}^{-2}$. A análise da competitividade foi realizada por meio de diagramas aplicados a experimentos substitutivos e índices de competitividade. A soja apresentou competitividade superior ao capim-pé-de-galinha para as variáveis de matéria seca, quando ambos estavam em proporções iguais de plantas.

Palavras-chave: Glycine max, Eleusine indica, competição, série de substituição.

\section{ABSTRACT}

The competition is a negative interference in which individuals compete for environmental resources. The objective was to evaluate the competitiveness of goosegrass with soybean. The experiment was conducted in green-house conditions, in a completely randomized design with four replications. The treatments were five proportions of crop and weed, respectively, 8:0, 6:2, 4:4, 2:6 and 0:8, which corresponded to 100,75, 50, 25 and $0 \%$ of plants soybean and opposite to the goosegrass, which constant population of eight plants per pot (8L), corresponding to approximately 250 plants $\mathrm{m}^{-2}$. The competitiveness analysis was accomplished through diagrams applied to replacement series experiments and competivity index. Soybean showed competitiveness superior to goosegrass for the variables of dry matter, when both were in similar proportions of plants.

Key words: Glycine max, Eleusine indica, competition, replacement series.

\section{INTRODUÇÃO}

A competição entre plantas é definida como o recrutamento conjunto de recursos essenciais ao crescimento, que são limitados no ecossistema comum (PITELLI, 1985). A importância do conhecimento dos efeitos competitivos deve-se ao fato de estarem diretamente ligados à melhor combinação entre as espécies envolvidas na associação (OLIVEIRA \& SCHREINER, 1987).

Adeterminação das interações competitivas entre plantas exige delineamentos experimentais e métodos apropriados, sendo os experimentos substitutivos convencionais os mais utilizados para esclarecer tais relações (FLECK et al., 2008). Esses modelos incluem análises de monoculturas, bem como misturas destas, em que as proporções das duas espécies estudadas variam, enquanto que a densidade total de plantas é mantida constante para todos os tratamentos (COUSENS, 1991; RADOSEVICH et al., 1997).

'Universidade de Passo Fundo (UPF), BR 285, São José, 99052-900, Passo Fundo, RS, Brasil. E-mail: alanacdw@hotmail.com. *Autor para correspondência. 
A espécie daninha Eleusine indica (L.) Gaertn. (capim-pé-de-galinha) é considerada importante gramínea infestante em mais de 50 culturas no mundo (RADOSEVICH et al., 1997; LEE \& NGIM, 2000). Considerando que essa espécie tem sido encontrada com frequência em lavouras do sul do Brasil, a investigação do potencial competitivo do capim-pé-de-galinha com a cultura da soja é de fundamental importância para o manejo dessa espécie. A hipótese da presente pesquisa é de que as plantas de soja são mais competitivas que a planta daninha, quando ambas estão em proporções iguais em uma mistura. Para isso, o objetivo deste trabalho foi investigar a competitividade do capim-pé-de-galinha com a cultura da soja, por meio de experimentos em série de substituição.

\section{MATERIAL E MÉTODOS}

O experimento foi realizado em casa-devegetação na estação de crescimento 2010/2011, em vasos plásticos com capacidade de $8 \mathrm{~L}$ e altura de $25 \mathrm{~cm}$ (unidades experimentais), preenchidos com solo da área experimental, classificado como Latossolo Vermelho Distrófico típico.

O delineamento foi inteiramente casualizado, com quatro repetições para cada tratamento. Os tratamentos consistiram de combinações de cinco proporções de soja e capimpé-de-galinha, ou seja, 8:0, 6:2, 4:4, 2:6 e 0:8, que corresponderam às proporções de 100:0, 75:25, 50:50, 25:75 e 0:100, respectivamente. A densidade populacional foi de oito plantas $\mathrm{vaso}^{-1}$, equivalente a aproximadamente 250 plantas $\mathrm{m}^{-2}$.

Após superação de dormência das sementes de capim-pé-de-galinha, elas foram postas em bandejas de isopor contendo 128 células e preenchidas com substrato comercial, e ali permaneceram até a emergência, que ocorreu 20 dias após a germinação. Em virtude da emergência, as plântulas de capim-péde-galinha foram transplantadas definitivamente para os vasos, coincidindo com a emergência de plântulas de soja, semeadas sete dias antes da implantação final das unidades experimentais, com a soja e o capim-péde-galinha, no mesmo estádio fenológico.

As variáveis analisadas nas plantas da cultura e da espécie daninha, aos 60 dias após a emergência da soja, foram: matéria seca da parte aérea, raiz e total. Para análise das variáveis, utilizouse o método da análise gráfica ou convencional para experimentos substitutivos (ROUSH et al., 1989; COUSENS, 1991), que consiste na construção de diagramas com base na produtividade relativa (PR) e produtividade relativa total (PRT), nas proporções de $0,25,50,75$ e $100 \%$ da cultura e da planta daninha.

A produtividade relativa foi calculada pela divisão da média da mistura pela média da monocultura, em cada variável analisada, incluindose no cálculo a média por planta de cada espécie em cada unidade experimental. A PRT representou a soma das produtividades relativas dos competidores nas respectivas proporções de plantas (HOFFMAN \& BUHLER, 2002).

Para o cálculo das produtividades relativas, utilizaram-se fórmulas de acordo com HOFFMAN \& BUHLER (2002), em que PRa = (p) (Amix/Amon); $\mathrm{PRb}=(1-\mathrm{p})(\mathrm{Bmix} / \mathrm{Bmon}) ; \mathrm{PRT}=\mathrm{PRa}+\mathrm{PRb}, \mathrm{em}$ que: $\mathrm{PRa}=$ produtividade relativa da espécie "a" (cultura); $\mathrm{PRb}=$ produtividade relativa da espécie "B" (daninha); $\mathrm{p}=$ proporção de "a" em \% dividido por 100; Amix = valor da variável a ser analisada (por exemplo: matéria seca) de "A" em mistura; Amon = valor da variável a ser analisada de " $A$ " em monocultura; Bmix = valor da variável a ser analisada de "B" em mistura; Bmon = valor da variável a ser analisada de "B" em monocultura; PRT = produtividade relativa total.

Os índices $\mathrm{CR}, \mathrm{K}$ e A foram calculados considerando a proporção de $50 \%$ de plantas de soja e de capim-pé-de-galinha. Dessa forma, CR representa o crescimento comparativo da espécie A em relação à espécie $\mathrm{B} ; \mathrm{K}$ indica a dominância relativa de uma espécie sobre a outra; e A aponta qual espécie é mais competitiva. A interpretação conjunta desses valores permite inferir o grau de competitividade entre as espécies com maior segurança (COUSENS, 1991). A espécie A é mais competitiva que a espécie $B$ quando $\mathrm{CR}>1, \mathrm{Ka}>\mathrm{Kb}$ e $\mathrm{A}>0$. Ao contrário, a espécie $B$ é mais competitiva que a espécie A quando $\mathrm{CR}<1$, $\mathrm{Ka}<\mathrm{Kb}$ e $\mathrm{A}<0$. As fórmulas para o cálculo dos índices de competição foram baseadas em HOFFMAN \& BUHLER (2002), em que: $C R=((1-p) / p)(P R a / P R b)$; $\mathrm{Ka}=((1-\mathrm{p}) / \mathrm{p})(\mathrm{PRa} /(10-\mathrm{PRa})) ; \mathrm{Kb}=((1-\mathrm{p}) / \mathrm{p})(\mathrm{PRb} /(1-$ $\mathrm{PRb})) ; \mathrm{A}=(\mathrm{PRa} / 2 \mathrm{p})-(\mathrm{PRb} /(2(1-))$.

Ciência Rural, v.43, n.12, dez, 2013. 
Para a análise estatística da produtividade relativa, calcularam-se primeiramente as diferenças para os valores de PR (DPR) obtidos nas proporções de 25,50 e $75 \%$ de plantas em relação aos valores pertencentes às retas hipotéticas nas respectivas proporções: $0,25,0,50$ e 0,75 . Utilizou-se o teste ' $t$ ' a $5 \%$ de probabilidade de erro para testar as diferenças relativas aos índices DPR, PRT, CR, K e A em relação às retas hipotéticas (HOFFMAN \& BUHLER, 2002), através do software estatístico SAS (Statistical Analysis System versão 8.0).

As hipóteses de nulidade para testar as diferenças de DPR e A eram de que as médias fossem iguais a zero $\left(\mathrm{H}_{0}=0\right)$; para PRT e $\mathrm{CR}$, que as médias fossem iguais à unidade $\left(\mathrm{H}_{0}=1\right)$; e, para o índice $\mathrm{K}$, de que as médias das diferenças entre $\mathrm{Ka}$ e $\mathrm{Kb}$ fossem iguais a zero $\left[\mathrm{H}_{0}=(\mathrm{Ka}-\mathrm{Kb})=0\right]$. As variáveis analisadas foram expressas em valores médios por planta e submetidos à análise de variância pelo teste $\mathrm{F}$ $(\mathrm{P} \leq 0,05)$. Se significativas, as médias dos tratamentos foram comparadas pelo teste de Dunnett $(\mathrm{P} \leq 0,05)$, considerando as monoculturas como testemunhas.

\section{RESULTADOS E DISCUSSÃO}

Os resultados obtidos mostraram valores de PR e PRT relativamente próximos aos valores hipotéticos quando há ausência de interferência de uma planta sobre a outra, para todas as variáveis analisadas (Figura 1). As variáveis matéria seca da parte aérea e total das plantas de soja e de capimpé-de-galinha não diferiram significativamente em nenhuma proporção de plantas, como pode ser observado na tabela 1. No entanto, pela análise gráfica, observou-se que a planta daninha obteve decréscimo na produtividade para essas variáveis, a partir da proporção 75:25. A reta da PRT declinou, apesar de não diferir significativamente pelo teste $t$ em nenhuma proporção de plantas, o que indica que a disponibilidade dos recursos foi suficiente para ambas as espécies (Figura 1 a, c; Tabela 1). Observou-se também que a matéria seca da parte aérea contribuiu mais para a matéria seca total do que a matéria seca da raiz, tendo em vista os valores relativamente semelhantes e os diagramas obtidos entre essas duas variáveis (Figura $1 \mathrm{a}, \mathrm{c}$ ).
Trabalhos sobre competição entre a cultura da soja e plantas daninhas demonstram que, em geral, as espécies daninhas gramíneas causam mais prejuízos à soja, comparadas a espécies daninhas dicotiledôneas (FLECK \& CANDEMIL, 1995). No entanto, as culturas podem representar uma menor habilidade competitiva, o que pode ser um efeito ocasionado pela densidade de plantas daninhas na área (BIANCHI et al., 2006).

A matéria seca da raiz de soja apresentou produtividade superior em relação à produtividade hipotética na ausência de competição, nas proporções 75:25 e 50:50. No entanto, tais diferenças só foram significativas na proporção 75:25. A matéria seca da raiz do capim-pé-de-galinha apresentou decréscimo em relação à produção hipotética na ausência de competição, nas proporções de 50:50 e 25:75, mas tais diferenças não foram significativas para essas proporções. A PRT indicou benefício para as espécies quando a soja estava em maior densidade e prejuízo mútuo quando a planta daninha estava em maior densidade, embora tais valores não diferiram significativamente da produtividade hipotética, quando não há interferência de uma espécie sobre a outra (Figura 1 b e Tabela 1).

A infestação de plantas daninhas durante o desenvolvimento inicial da cultura da soja representa importante problema para as lavouras agrícolas, pois resulta em perdas significativas no rendimento de grãos, dependendo da composição específica da comunidade infestante e da habilidade competitiva das espécies em convivência (DIAS et al., 2010). LAMEGO et al. (2005) destacam que a soja é uma espécie que apresenta elevada plasticidade e mostra respostas adaptativas às condições de ambiente e manejo ao qual é submetida. Dessa forma, pode não ser influenciada pela competição com algumas espécies de plantas daninhas, como foi verificado neste trabalho para as variáveis de matéria seca. Em estudo onde o capim-pé-de-galinha conviveu com a cultura do milho, observou-se habilidade competitiva equivalente entre as duas espécies em relação às variáveis matéria seca da parte aérea, raiz e total (WANDSCHEER et al., 2013). 


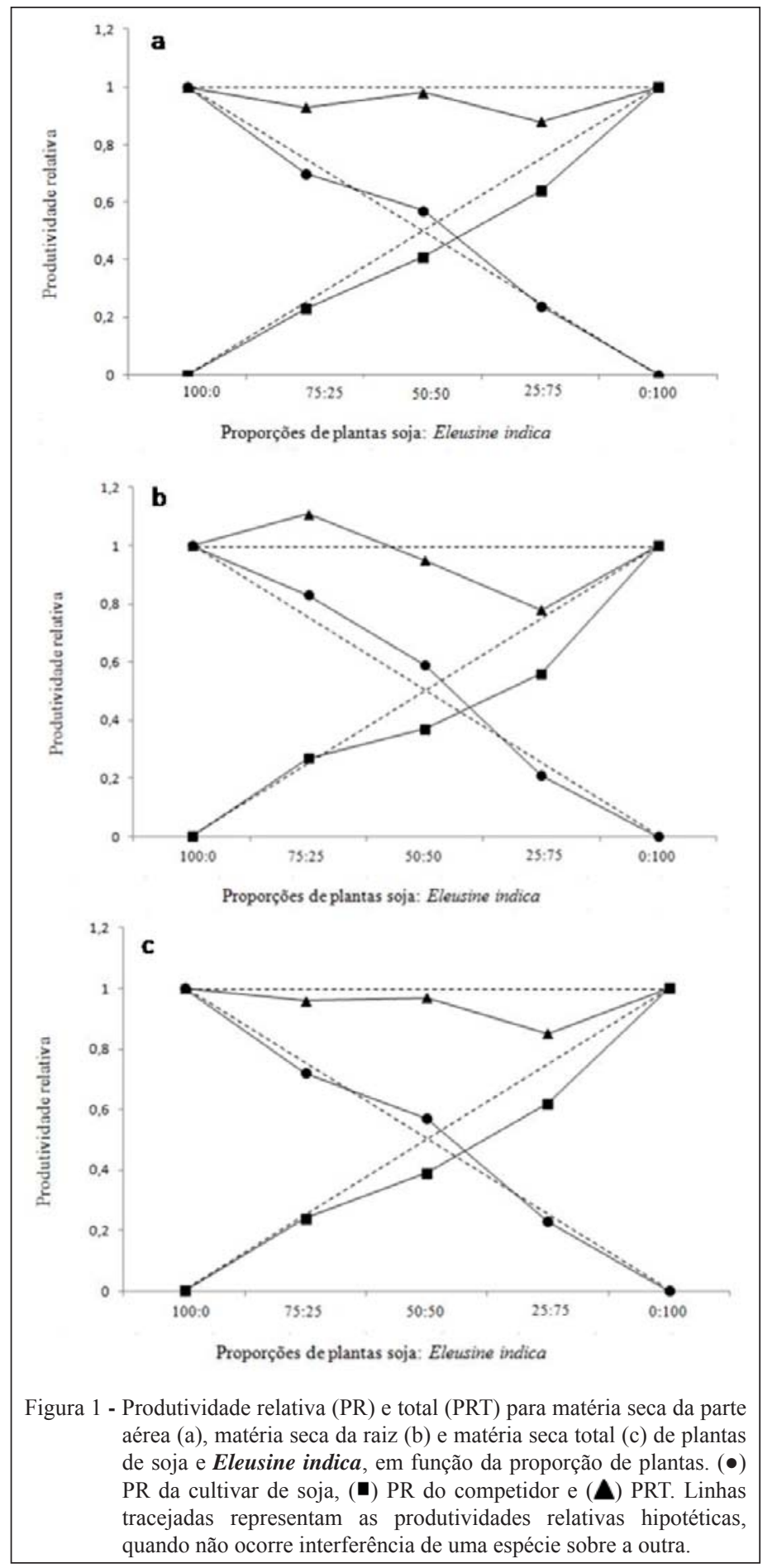

A habilidade da soja em competir com as plantas daninhas, especialmente no período vegetativo, garante a esta vantagem na busca por recursos do meio e na síntese de fotoassimilados, que serão utilizados para o enchimento de grãos no período reprodutivo (LAMEGO et al., 2004).
Os índices de competitividade (CR, K e A) expressam o quanto uma espécie é mais competitiva que a outra, quando em proporções iguais. Para os experimentos de competição de soja e capim-péde-galinha, quando as duas espécies estiveram nas mesmas proporções, pode-se observar que não

Ciência Rural, v.43, n.12, dez, 2013. 
Tabela 1 - Diferenças relativas de produtividade (DPR) para as variáveis matéria seca da parte aérea, raiz e total; e produtividade relativa total (PRT), nas proporções 75/25, 50/50 e 25/75 de plantas de soja associadas com capim-pé-de-galinha.

\begin{tabular}{|c|c|c|c|}
\hline & $75 / 25$ & $50 / 50$ & $25 / 75$ \\
\hline \multicolumn{4}{|l|}{ MSPA $^{1}$} \\
\hline DPR soja & $-0,05( \pm 0,09)^{\mathrm{ns}}$ & $0,07( \pm 0,03)^{\mathrm{ns}}$ & $-0,01( \pm 0,02)^{\mathrm{ns}}$ \\
\hline DPR capim pé-de-galinha & $-0,03( \pm 0,03)^{\mathrm{ns}}$ & $-0,09( \pm 0,06)^{\mathrm{ns}}$ & $-0,18( \pm 0,19)^{\mathrm{ns}}$ \\
\hline PRT & $0,93( \pm 0,12)^{\mathrm{ns}}$ & $0,98( \pm 0,05)^{\mathrm{ns}}$ & $0,88( \pm 0,10)^{\mathrm{ns}}$ \\
\hline \multicolumn{4}{|l|}{$\mathrm{MSR}^{2}$} \\
\hline DPR soja & $0,08( \pm 0,02) *$ & $0,09( \pm 0,04)^{\mathrm{ns}}$ & $-0,03( \pm 0,04)^{\mathrm{ns}}$ \\
\hline DPR capim pé-de-galinha & $0,03( \pm 0,10)^{\mathrm{ns}}$ & $-0,13( \pm 0,08)^{\mathrm{ns}}$ & $-0,19( \pm 0,10)^{\mathrm{ns}}$ \\
\hline PRT & $1,11( \pm 0,10)^{\mathrm{ns}}$ & $0,96( \pm 0,11)^{\mathrm{ns}}$ & $0,78( \pm 0,11)^{\mathrm{ns}}$ \\
\hline \multicolumn{4}{|l|}{$\mathrm{MST}^{3}$} \\
\hline DPR soja & $-0,03( \pm 0,08)^{\mathrm{ns}}$ & $0,07( \pm 0,03)^{\mathrm{ns}}$ & $-0,02( \pm 0,02)^{\mathrm{ns}}$ \\
\hline DPR capim pé-de-galinha & $-0,01( \pm 0,06)^{\mathrm{ns}}$ & $-0,11( \pm 0,06)^{\mathrm{ns}}$ & $-0,13( \pm 0,10)^{\mathrm{ns}}$ \\
\hline PRT & $0,96( \pm 0,13)^{\mathrm{ns}}$ & $0,97( \pm 0,06)^{\mathrm{ns}}$ & $0,85( \pm 0,10)^{\mathrm{ns}}$ \\
\hline
\end{tabular}

${ }^{\text {ns }}$ Não significativo ${ }^{*}$ significativo pelo teste $\mathrm{t}(\mathrm{P} \leq 0,05)$. Valores entre parênteses representam o erro-padrão da média. ${ }^{1} \mathrm{MSPA}$ : matéria seca da parte aérea; ${ }^{2} \mathrm{MSR}$ : matéria seca da raiz; ${ }^{3} \mathrm{MST}$ : matéria seca total (raiz + parte aérea).

houve diferenças significativas para os índices $\mathrm{CR}$ e A nas variáveis matéria seca da parte aérea e total. Entretanto, o valor de $\mathrm{K}$ diferiu para essas variáveis, o que significa que a soja domina em relação ao capim-pé-de-galinha $(\mathrm{Ka}>\mathrm{Kb})$. Para a variável matéria seca da raiz, apenas o índice A foi significativo e indicou ser a soja mais agressiva com relação ao sistema radical, comparada à planta daninha $(\mathrm{A}>0)$ (Tabela 2).

Em análise à resposta da soja com a interferência do capim-pé-de-galinha, constatouse que as variáveis matéria seca da parte aérea, raiz e total não diferiram dos valores obtidos nos monocultivos (Tabela 3). O contrário foi observado por CARVALHO et al. (2011), quando o capimmarmelada foi mais sensível que a cultura do trigo em relação à competição intraespecífica. Também,
AMINPANAH et al. (2012) constataram que o arroz (cultivar 'Hashemi') apresentou melhor peso da matéria seca da parte aérea quando conviveu com plantas da mesma espécie.

Com base nos resultados obtidos no presente estudo, pode-se constatar que a soja é mais competitiva que o capim-pé-de-galinha em relação às variáveis de matéria seca, quando ambas as espécies encontram-se em proporções iguais em uma mistura, o que confirma a hipótese deste trabalho.

\section{AGRADECIMENTOS}

À Coordenação de Aperfeiçoamento de Pessoal de Nível Superior (CAPES), pela bolsa de pesquisa concedida ao primeiro autor, e à Universidade de Passo Fundo (UPF), através do Programa de Pós-graduação em Agronomia, pela infraestrutura necessária à pesquisa.

Tabela 2 - Índices de competitividade de soja e capim-pé-de-galinha, expressos por competitividade relativa (CR), coeficientes de agrupamento relativo $(\mathrm{K})$ e agressividade $(\mathrm{A})$.

\begin{tabular}{lcccc}
\hline Variáveis & $\mathrm{CR}$ & $\mathrm{K}_{\mathrm{s}=\text { soja }}$ & $\mathrm{K}_{\mathrm{c}=\text { capim-pé-de-galinha }}$ & $\mathrm{A}$ \\
\hline MSPA $^{1}$ & $1,49( \pm 0,24)^{\mathrm{ns}}$ & $1,35( \pm 0,13)^{*}$ & $0,74( \pm 0,18)^{*}$ & $0,16( \pm 0,07)^{\mathrm{ns}}$ \\
MSR $^{2}$ & $1,80( \pm 0,33)^{\mathrm{ns}}$ & $1,52( \pm 0,31)^{\mathrm{ns}}$ & $0,65( \pm 0,20)^{\mathrm{ns}}$ & $0,22( \pm 0,06)^{*}$ \\
MST $^{3}$ & $1,58( \pm 0,28)^{\mathrm{ns}}$ & $1,36( \pm 0,12)^{*}$ & $0,70( \pm 0,18)^{*}$ & $0,18( \pm 0,08)^{\mathrm{ns}}$ \\
\hline
\end{tabular}

${ }^{n s}$ Não-significativo e ${ }^{*}$ significativo pelo teste $\mathrm{t}(\mathrm{P} \leq 0,05)$. Valores entre parênteses representam o erro-padrão da média. ${ }^{1} \mathrm{MSPA}$ : matéria seca da parte aérea; ${ }^{2} \mathrm{MSR}$ : matéria seca da raiz; ${ }^{3} \mathrm{MST}$ : matéria seca total (raiz + parte aérea).

Ciência Rural, v.43, n.12, dez, 2013. 
Tabela 3 - Resposta da soja à interferência com o capim-pé-de-galinha, aos 60 dias após a emergência.

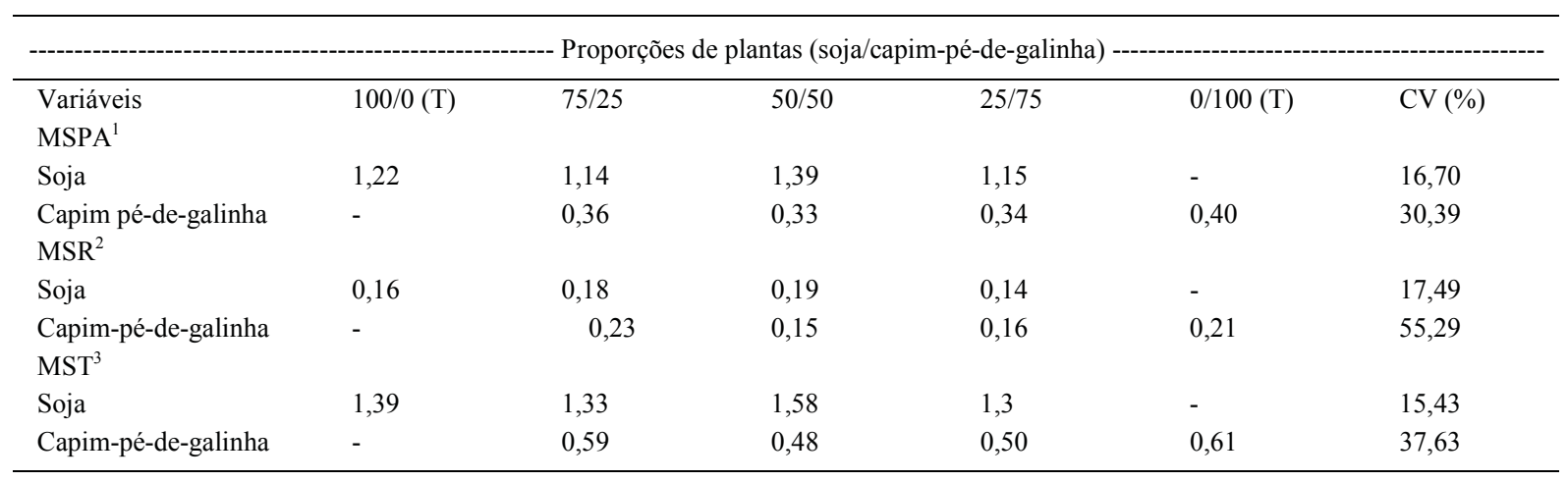

* Média difere da testemunha (T) pelo teste de Dunnett $(\mathrm{P} \leq 0,05)$. CV = coeficiente de variação. ${ }^{1} \mathrm{MSPA}$ : matéria seca da parte aérea; ${ }^{2} \mathrm{MSR}$ : matéria seca da raiz; ${ }^{3} \mathrm{MST}$ : matéria seca total (raiz + parte aérea).

\section{REFERÊNCIAS}

AMINPANAH, $\mathrm{H}$. et al. Interference interactions of two species of Echinochloa genus with rice. Chilean Journal of Agricultural Research, v.72, n.3, p.364-370, 2012. Disponível em: <http:// www.scielo.cl/scielo.php?pid=S071858392012000300010\&scrip $\mathrm{t}=\mathrm{sci}$ arttext\&tlng=pt $>$. Acesso em: 20 maio 2013. doi: 10.4067/ S0718-58392012000300010.

BIANCHI, M.A.; et al. Proporção entre plantas de soja e plantas competidoras e as relações de interferência mútua. Ciência Rural, v.36, n.5, p.1380-1387, 2006. Disponível em: <http://www.scielo. br/scielo.php?script $=$ sci_arttext\&pid $=$ S0103847820060005000 06\&lng $=\mathrm{pt \& nrm}=\mathrm{iso} \& \mathrm{tlng}=\mathrm{pt}>$. Acesso em: 10 nov. 2011. doi: 10.1590/S0103-84782006000500006.

CARVALHO, L.B. de et al. Effects of plant density and proportion on the interaction between wheat with alexandergrass plants. Bragantia, v.70, n.1, p.40-45, 2011. Disponível em: < http://www. scielo.br/scielo.php?script $=$ sci arttext\&pid $=\mathrm{S} 000687052011000$ 100002\&lng=en\&nrm=iso\&tlng=pt $>$. Acesso em: 20 dez. 2011. doi: 10.1590/S000687052011000100002.

COUSENS, R. Aspects of the design and interpretation of competition (interference) experiments. Weed Technology, v.5, n.3, p.664-673, 1991. Disponível em: <http://www.jstor.org/disc over $/ 10.2307 / 3987056$ ?uid=3737664\&uid=2129\&uid=2134\&uid $=2 \&$ uid $=70 \&$ uid $=4 \&$ sid $=21102572457973>$. Acesso em: $22 \mathrm{dez}$. 2011.

DIAS, A.C.R. et al. Competitiveness of Alexandergrass or Bengal Dayflower with soybean. Planta Daninha, v.28, n.3, p.515-522, 2010. Disponível em: $<$ http://www.scielo.br/scielo.php?script=sci arttext\&pid $=$ S0100-83582010000300008 $>$. Acesso em: 20 maio 2013. doi: 10.1590/S0100-83582010000300008.

FLECK, N.G.; CANDEMIL, C.R.G. Interferência de plantas daninhas na cultura da soja (Glycine max (L.) Merrill). Ciência Rural, v.25, n.1, p.27-32, 1995. Disponível em: <http://www. scielo.br/pdf/cr/v25n1/a06v25n1.pdf>. Acesso em: 15 jul. 2010.

FLECK, N.G. et al. Competitividade relativa entre cultivares de arroz irrigado e biótipo de arroz-vermelho. Planta Daninha, v.26, n.1, p.101-111, 2008. Disponível em: <http://www.scielo.br/scielo.
php?script=sci_arttext\&pid=S010083582008000100011\&lng= en\&nrm $=$ iso \&tlng=pt $>$. Acesso em: 06. jan. 2011. doi: 10.1590/ S0100-83582008000100011.

HOFFMAN, M.L.; BUHLER, D.D. Utilizing Sorghum as a functional model of crop-weed competition. I. Establishing a competitive hierarchy. Weed Science, v.50, n.4, p.466-472, 2002. Disponível em: <http://www.jstor.org/discover/10.2307/4046528>. Acesso em: 06. jan. 2011.

LAMEGO, F.P. et al. Tolerância à interferência de plantas competidoras e habilidade de supressão por genótipos de soja - II. Resposta de variáveis de produtividade. Planta Daninha, v.22, n.4, p.491-498, 2004. Disponível em: <http://www.scielo.br/scielo. php? script $=$ sci arttext\&pid $=$ S010083582004000400002\&lng $=$ en\&nrm=iso\&tlng=pt $>$. Acesso em: 12 jul. 2012. doi: 10.1590/ S0100-83582004000400002.

LAMEGO, F.P. et al. Tolerância à interferência de plantas competidoras e habilidade de supressão por cultivares de soja I. Resposta de variáveis de crescimento. Planta Daninha, v.23, n.3, p.405-414, 2005. Disponível em: <http://www.scielo.br/scielo. php script $=$ sci_arttext\&pid $=$ S0 $10083582005000300003 \& \operatorname{lng}=$ en\&nrm=iso\&tlng=pt $>$. Acesso em: 12 jul. 2012. doi: 10.1590/ S0100-83582005000300003

LEE, L.J.; NGIM, J. A first report of glyphosate-resistant goosegrass (Eleusine indica (L) Gaertn) in Malaysia. Pest Management Science, v.56, n.1, p.36-339, 2000. Disponível em: $<$ http://onlinelibrary.wiley.com/doi/10.1002/(SICI)15264998(200 004)56:4\%3C336::AIDPS123\%3E3.0.CO;28/abstract;jsessionid= 39920B5FAB877E7FA623A5FAB953FEC7.d03t04?deniedAcces sCustomisedMessage $=\&$ userIs Authenticated $=$ false $>$. Acesso em: 05 jul. 2010.

OLIVEIRA, E.B. de; SCHREINER, H.G. Caracterização e análise estatística de experimentos de agrossilvicultura. Boletim de Pesquisa Florestal, v.1, n.15, p.19-40, 1987. Disponível em: <http://www.cnpf.embrapa.br/publica/boletim/boletarqv/boletim15/ oliveira.pdf>. Acesso em: 05 jul. 2010.

PITELLI, R.A. Interferência de plantas daninhas em culturas agrícolas. Informe Agropecuário, v.11, n.129, p.16-25, 1985. Disponível em: <http://www.ipef.br/PUBLICACOES/stecnica/ nr12/cap01.pdf>. Acesso em: 05 jul. 2010. 
RADOSEVICH, S.R. et al. Plant-plant associations. In: RADOSEVICH, S.R.; et al. Ecology of weeds and invasive plants: relationship to agriculture and natural resource management. 3.ed. New Jersey: John Wiley \& Sons, 1997. 454p.

ROUSH, M.L. et al. A comparison of methods for measuring effects of density and proportion in plant competition experiments.
Weed Science, v.37, n.2, p.268-275, 1989. Disponível em: <http:// www.jstor.org/stable/4044855>. Acesso em: 17 jun. 2010.

WANDSCHEER, A.C.D. et al. Competitive ability of corn in coexistence with goosegrass. Planta Daninha, v.31, n.2, p.281289, 2013. Disponível em: $<$ http://www.scielo.br/scielo.php?pid= $\mathrm{S} 010083582013000200005 \&$ script $=$ sci_arttext\&tlng $=\mathrm{pt}>$. Acesso em: 20 maio 2013. doi: 10.1590/S0100-83582013000200005. 